\title{
Shared roles of dorsal and subgenual anterior cingulate cortices in economic decisions
}

Habiba Azab and Benjamin Y. Hayden

*Corresponding author:

Habiba Azab

15

Department of Brain and Cognitive Sciences

16 University of Rochester

17

Rochester NY 14618

18 


\section{ABSTRACT}

21 Theories of dorsal anterior cingulate cortex (dACC) function generally emphasize

22 its cognitive and regulatory functions while theories of subgenual ACC (sgACC)

23 emphasize its emotional, limbic, and arousal-related roles. But how different are these

24 areas when compared in the same task? We recorded neuronal responses in both regions

25 in macaques in a task with cognitive and limbic aspects, a token gambling task. Using

26 tokens allowed us to compare responses to wins and losses. Both regions phasically

27 encoded several important economic variables in similar ways; these included offered

28 values, remembered values, attended values, and obtained values, and number of current

29 tokens. Signal-to-noise ratio in $\operatorname{sgACC}$ was substantially lower than in $\mathrm{dACC}$, and

30 sgACC neurons responded more strongly to losses and in anticipation of large rewards.

31 These results highlight the common economic functions of the anterior cingulum and

32 suggest different functional emphases between regions, rather than a strict cognitive vs.

33 emotional division. 


\section{INTRODUCTION}

41 The cingulum is a large structure on the medial wall encircling the corpus

42 callosum in the saggital plane. The portion rostral to the central sulcus, the anterior

43 cingulate cortex (ACC), is important for almost every aspect of cognition and its

44 malfunction is associated with several psychiatric diseases (Bush et al., 1999;

45 Heilbronner \& Hayden, 2016; Paus, 2001; Rushworth et al., 2012; Shenhav et al., 2013).

46 A great deal of evidence indicates that the ACC can be functionally subdivided into at

47 least two portions, a dorsal (dACC) and subgenual ( $\operatorname{sg} \mathrm{ACC})$ one. These portions are

48 associated with different cytoarchitectonics, connectivity patterns, patterns of

49 hemodynamic activation, and lesion effects (Bush et al., 2000; Vogt et al., 1995).

50 Nonetheless, a direct comparison of their function at the single-neuron level has never

51 been made.

52 Theories of dACC generally emphasize its cognitive and regulatory roles rather

53 than limbic and emotional ones. Cognitive functions associated with dACC include error-

54 and conflict-monitoring, allocation of control, task-switching and regulation of strategy

55 (Allman et al., 2001; Amiez et al., 2005; Carter et al., 2007; Kerns et al., 2004; Neubert et

56 al., 2015; Procyk et al., 2000; Quilodran et al., 2008; Rushworth et al., 2002; Shenhav et

57 al., 2013). In contrast, theories of sgACC emphasize its limbic, emotional, and arousal-

58 related roles and not its cognitive function. These functions include emotional processing,

59 mood-changes, sleep, and potentially a role in depression (Botteron et al., 2002; Coryell

60 et al., 2005; Drevets et al., 1997; George et al., 2006; Johansen-Berg et al., 2008; Rolls et

61 al., 2003). Some evidence suggests that these regions have negatively correlated activity

62 patterns (Bush et al., 2000), consistent with the idea they inhibit each other. These 
63 differences have led to the theory that these two regions play antagonistic roles in

64 cognition (Bush et al., 2000).

65 Nonetheless, there are reasons to believe that dACC and sgACC play overlapping

66 roles in cognition. Both are associated with monitoring and anticipation of reward (which

67 itself has both cognitive and limbic aspects.) The role of the dorsal portion of ACC in

68 reward representation is well-established (Heilbronner and Hayden, 2016). Although

69 single-unit studies in the subgenual portion have been relatively few, some of these

70 studies also implicate this region in reward processing and representation (Monosov \&

71 Hikosaka, 2012; Rudebeck et al., 2014; similar findings were reported in Amemori \&

72 Graybiel, 2012, who recorded in the adjacent pregenual cingulate). More broadly, both

73 regions have been implicated in pain processing (Derbyshire et al., 1998; Vogt et al.,

74 1992), depression (Cotter et al., 2001; Mayberg et al., 1997), addiction (Forman et al.,

75 2004; Goldstein et al., 2007), OCD (Graybiel \& Rauch, 2000), and other psychiatric

76 disorders (Blumberg et al., 2000; Bouras et al., 2001; Drevets et al., 1997; Drevets et al.,

77 2008; Ongur, Drevets \& Price, 1998). Nonetheless, our ability to compare these regions

78 is limited by the paucity of direct comparisons of their function.

79 Here we compared the responses of these two regions in a single gambling task.

80 We sought to understand their relative contributions within a single task that was

81 complex and engaging, and had both limbic and cognitive aspects. Among economic

82 choice tasks, gambling is particularly useful because it is well-characterized

83 mathematically, preferences are stable, and it involves both reward and

84 monitoring/adjustment processes (Heilbronner and Hayden, 2013; Heilbronner \&

85 Hayden, 2015). Furthermore, we have a foundational understanding of the neuroscience 
86 of risky choice, including recordings in AACC, although not in sgACC (Blanchard and

87 Hayden, 2014; Hayden et al., 2011; Hosokawa et al., 2013; Kennerley et al., 2009;

88 Procyk et al., 2000). To directly compare coding of gain and loss, we added a new feature

89 to our task. On each trial, monkeys gambled for gain or loss of tokens; collection of 6

90 tokens produced a jackpot reward (cf. Seo \& Lee, 2007). This feature allowed for direct

91 comparison of two reward signs in a single modality (thus controlling for sensory

92 features that, say, an air puff or hypertonic solution would not). This manipulation also

93 allowed us to explore responses to secondary reinforcers - which has not been done in

94 sgACC.

95 These two cingulate regions exhibited strikingly similar patterns of activity in our

96 study. Both encoded several economic variables and did so in similar ways. Specifically,

97 we found encoding of the values of offers (attended and remembered), outcomes, and

98 number of tokens accumulated. Both regions used an attentionally-aligned, as opposed to

99 labeled line, coding scheme for values. We also confirmed earlier results showing

100 encoding of positions of offered and chosen options (Strait et al., 2016). We found no

101 measurable differences in response latency. This is not to say the regions were identical:

102 two major differences stood out. First, task-relevant coding was more frequent in dACC

103 and signal-to-noise was roughly three times greater. Second, neurons in dACC were

104 biased towards higher firing for gamble wins, while neurons in sgACC were biased

105 towards higher firing for losses. There was also a positive bias at the single neuron level

106 in anticipation of large, primary rewards in sgACC; no corresponding bias occurred in

$107 \mathrm{dACC}$. These data suggest that $\mathrm{dACC}$ and sgACC play overlapping roles in economic 
108 decisions albeit with different emphases, but do not have antagonistic roles or strong

109 cognitive / emotional specialization.

110

111

A
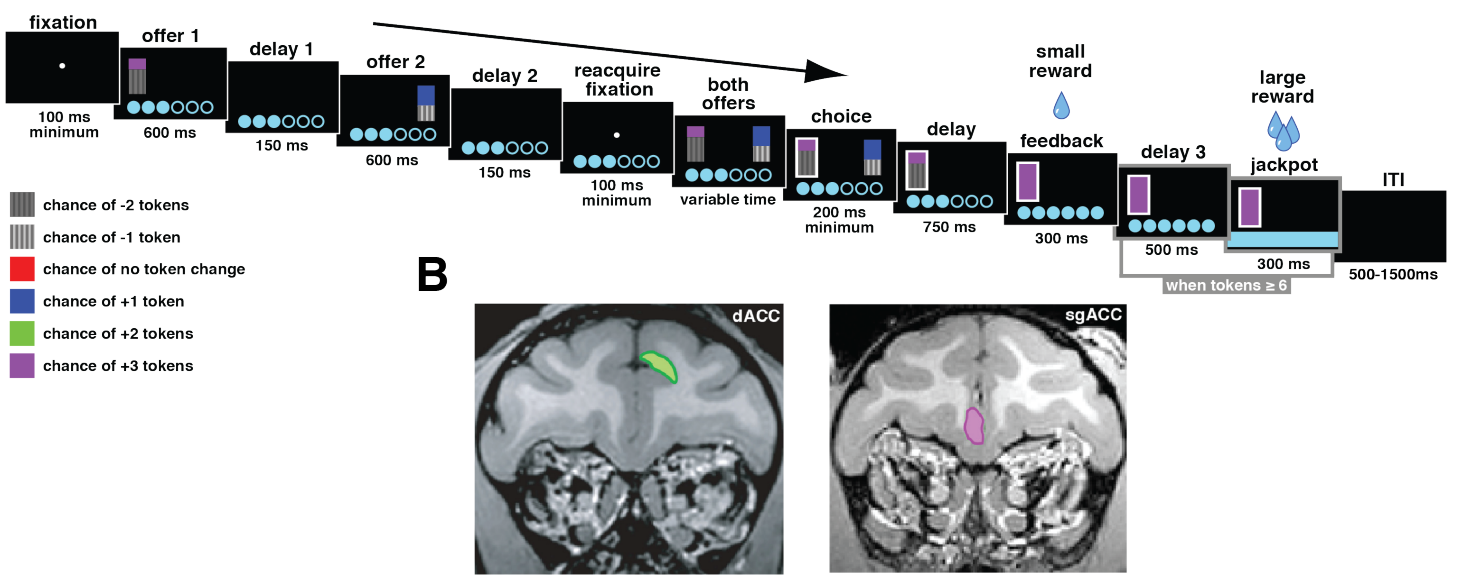

113 Figure 1: A: Example trial from token-gambling task. Offers were presented

114 asynchronously, and the side the first offer appeared on was counterbalanced across

115 trials. Each gamble offered one of two outcomes (indicated by the colors of the bars) at a

116 certain probability (indicated by their heights). B: Regions of interest (for exact

117 coordinates, see Methods).

118

A

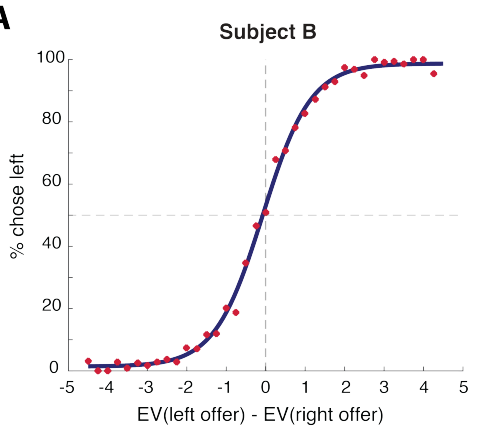

B

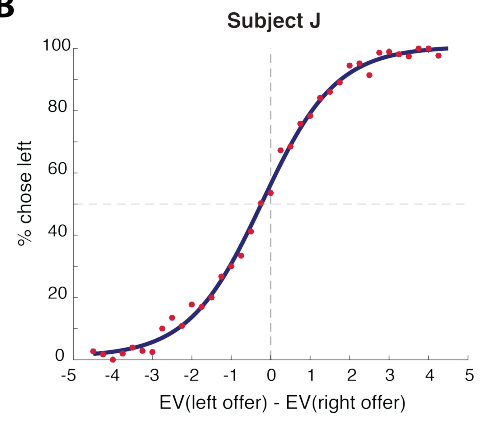

120 Figure 2: Behavior for each subject, fit to a sigmoid function. Subjects choose the left option more often as its value increases. EV: expected value of gamble. 


\section{RESULTS}

\section{Behavior}

126 Two monkeys performed a token-gambling task. On each trial, monkeys chose

127 between two options presented asynchronously that differed in three dimensions: win

128 amount, loss amount, and probability of win (Figure 1A). Both monkeys showed

129 behavior consistent with task understanding (Figure 2). Specifically, both subjects

130 preferred the option with the greater expected value (subject B: $80.3 \%$; subject J: 75.1\%;

131 both $\mathrm{P}<0.0001$; two-sided binomial test), and were sensitive to the three parameters that

132 defined each gamble (See Table 1). Subjects were more sensitive to win amount than to

133 loss amount, consistent with the idea that they focus on the win (Hayden \& Platt, 2007;

134 Hayden et al., 2008). Monkeys showed weak side and order biases (rightward choice:

$13551.0 \%$ in subject $\mathrm{B} ; 47.4 \%$ in subject $\mathrm{J}$; chose second: $52.5 \%$ in subject $\mathrm{B} ; 55.6 \%$ in

136 subject J.)

137 Monkeys were risk-seeking, consistent with earlier studies (Hayden et al., 2008;

138 Hayden \& Platt, 2007; McCoy \& Platt, 2005). To assess risk-seeking, we fit a utility

139 curve distortion parameter to subjects' behavior in each session (Lak et al., 2014;

140 Yamada et al., 2013). This parameter, $\alpha$, was always greater than 1 in both subjects (B: $\alpha$

$141=1.61(\mathrm{n}=66$ sessions $)$; subject $\mathrm{J}: 1.81(\mathrm{n}=74$ sessions $)$; both greater than 1 at $\mathrm{P}<$

1420.0001 ; one-sample t-test over sessions). Subjects' behavior was modestly influenced by

143 the number of current tokens: accuracy improved as tokens increased (subject B: $\beta=$

$1440.027, \mathrm{P}=0.0012$; subject $\mathrm{J}: \beta=0.078, \mathrm{P}<0.0001$; logistic regression of accuracy

145 against tokens acquired). 


\section{7}

\section{3}

157 the average fraction of variance in firing rates explained by this model for each neuron in

158 each brain region over the entire trial. We next compared these measures across regions,

159 where neurons were the unit of analysis. Task-relevant variables explained over three

160 times as much variance in $\mathrm{dACC}$ than in $\mathrm{sgACC}$ neurons (average adjusted $\mathrm{R}^{2}$ over all

161 neurons: dACC: 0.0093; sgACC: 0.0025; $\mathrm{T}=4.94, \mathrm{P}<0.0001$; two-sample t-test). Thus,

162 variance explained was between 3 and 4 times greater in $\mathrm{AACC}$ than in sgACC.

Table 1: Regression coefficients from a logistic regression model of choice (left $=1$, right $=0$ ) against the variables in the regressor column. A 'loss' within a gamble was always less than or equal to the win outcome of that gamble. Note that losses included negative outcomes. Overall, the expected value of the win-portion of a gamble influenced behavior more than the value of a loss did in both subjects.

\section{Variance explained by task variables is higher in dACC than in $\operatorname{sgACC}$}

$$
\text { Responses to task variables were attenuated in sgACC neurons compared to }
$$
dACC neurons. To quantify this difference, we regressed average firing rate in a sliding $500 \mathrm{~ms}$ window against eight task-relevant variables (see Methods). We then computed

\begin{tabular}{|c|r|c|}
\hline Regressor & Subject B & Subject J \\
\hline Win amount, left offer & $1.2302 \quad(\mathrm{P}<0.0001)$ & $1.0097 \quad(\mathrm{P}<0.0001)$ \\
\hline Loss amount, left offer & $0.4063 \quad(\mathrm{P}<0.0001)$ & $0.2451 \quad(\mathrm{P}<0.0001)$ \\
\hline Probability of win, left offer & $3.3828 \quad(\mathrm{P}<0.0001)$ & $3.1745 \quad(\mathrm{P}<0.0001)$ \\
\hline Win amount, right offer & $-1.1577 \quad(\mathrm{P}<0.0001)$ & $-1.1390 \quad(\mathrm{P}<0.0001)$ \\
\hline Loss amount, right offer & $-0.3449(\mathrm{P}<0.0001)$ & $-0.1937 \quad(\mathrm{P}<0.0001)$ \\
\hline Probability of loss, right-offer & $-4.1934 \quad(\mathrm{P}<0.0001)$ & $-3.5026 \quad(\mathrm{P}<0.0001)$ \\
\hline Intercept & $-0.2893 \quad(\mathrm{P}<0.0001)$ & $0.6177 \quad(\mathrm{P}<0.0001)$ \\
\hline
\end{tabular}




\begin{tabular}{|c|c|c|}
\hline Variable & dACC (t-statistic) & sgACC (t-statistic) \\
\hline Offer 1 value (attended) & $0.64(\mathrm{P}=0.53)$ & $1.67(\mathrm{P}=0.098)$ \\
\hline Offer 1 value (remembered) & $-0.26(\mathrm{P}=0.79)$ & $-0.47(\mathrm{P}=0.64)$ \\
\hline Offer 2 value (attended) & $0.95(\mathrm{P}=0.34)$ & $0.97(\mathrm{P}=0.33)$ \\
\hline Chosen value & $-1.47(\mathrm{P}=0.14)$ & $\mathbf{- 2 . 3 1}(\mathbf{P}=\mathbf{0 . 0 2 2})$ \\
\hline Unchosen value & $\mathbf{2 . 7 0}(\mathbf{P}=\mathbf{0 . 0 0 7 8})$ & $1.73(\mathrm{P}=0.085)$ \\
\hline Outcome & $\mathbf{2 . 3 5}(\mathbf{P}=\mathbf{0 . 0 2})$ & $\mathbf{- 2 . 8 5}(\mathbf{P}=\mathbf{0 . 0 0 5 0})$ \\
\hline Number of tokens & $1.42(\mathrm{P}=0.16)$ & $0.15(\mathrm{P}=0.88)$ \\
\hline Jackpot & $0.092(\mathrm{P}=0.93)$ & $\mathbf{4 . 4 7}(\mathbf{P}<\mathbf{0 . 0 0 0 1})$ \\
\hline
\end{tabular}

Table 2: Assessment of biases in tuning in the overall population. We performed this analysis running a one-sample t-test on the regression coefficients corresponding to each variable obtained from a multiple linear regression (see Methods).

\begin{tabular}{|c|c|c|}
\hline Variable & dACC (\% positive) & sgACC (\% positive) \\
\hline Offer 1 value (attended) & $57.6 \%(\mathrm{n}=19 / 33, \mathrm{P}=0.49)$ & $66.7 \%(\mathrm{n}=12 / 18, \mathrm{P}=0.24)$ \\
\hline Offer 1 value (remembered) & $56.0 \%(\mathrm{n}=14 / 25, \mathrm{P}=0.69)$ & $41.7 \%(\mathrm{n}=5 / 12, \mathrm{P}=0.77)$ \\
\hline Offer 2 value (attended) & $50.0 \%(\mathrm{n}=9 / 18, \mathrm{P}=1)$ & $57.1 \%(\mathrm{n}=8 / 14, \mathrm{P}=0.79)$ \\
\hline Chosen value & $20 \%(n=4 / 20, P=0.012)$ & $22.2 \%(\mathrm{n}=2 / 9, \mathrm{P}=0.18)$ \\
\hline Unchosen value & $\begin{array}{c}100 \%(\mathrm{n}=12 / 12, \mathrm{P}= \\
0.0005)\end{array}$ & $50.0 \%(\mathrm{n}=3 / 6, \mathrm{P}=1.0)$ \\
\hline Outcome & $\begin{array}{c}71.0 \%(n=22 / 36, P= \\
0.029)\end{array}$ & $31.6 \%(\mathrm{n}=6 / 19, \mathrm{P}=0.17)$ \\
\hline Number of tokens & $60.0 \%(\mathrm{n}=27 / 49, \mathrm{P}=0.23)$ & $58.3 \%(\mathrm{n}=14 / 31, \mathrm{P}=0.54)$ \\
\hline Jackpot & $54.3 \%(\mathrm{n}=19 / 35, \mathrm{P}=0.74)$ & $70.6 \%(\mathrm{n}=12 / 17, \mathrm{P}=0.14)$ \\
\hline
\end{tabular}

172 determine whether the fraction of positively tuned neurons (of the tuned population only)

173 is significantly larger than chance (50\%), using a two-sided binomial test. 
A

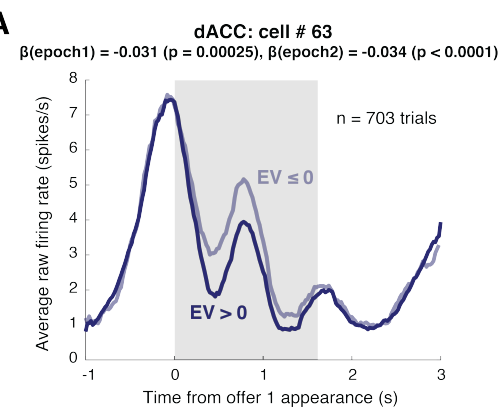

B

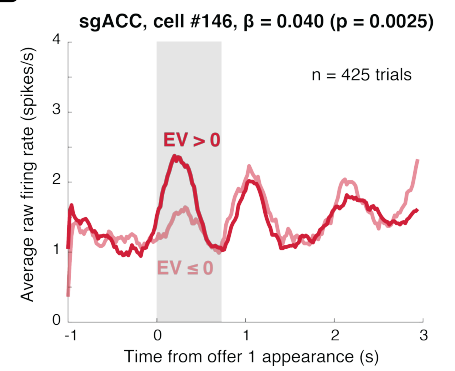

C

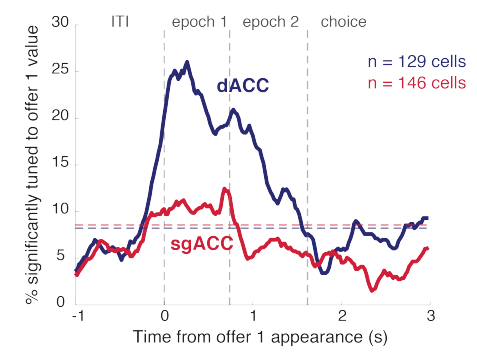

176 Figure 3: Neural responses to the value of offer 1. A: Responses of one dACC neuron

177 with increasing firing rate in response to smaller values of offer 1 in both the first and

178 second epochs. Shaded regions are epochs of interest with significant modulation. B:

179 Responses of one sgACC neuron with increasing firing rates in response to larger values

180 of offer 1 in the first epoch. C: Proportion of neurons selective for the value of offer $1 \mathrm{in}$

181 both regions throughout the course of the trial. This fraction was computed by regressing

182 firing rate over a $500 \mathrm{~ms}$ sliding window against the expected value of the first offer,

183 along with other task-relevant variables (see Methods). The blue and red horizontal lines

184 indicate the percentage of neurons we would expect to see by chance (as determined by a

185 two-sided binomial test, according to the number of neurons in each area) for dACC and

186 sgACC, respectively.

187

188 Neurons in both regions encode offered values

189 Responses of two neurons (one in $\mathrm{dACC}$ and one in sgACC) are shown in Figure

190 3. Both of these neurons' firing rates were correlated with the expected value of the first

191 offer while it was displayed on the screen (epoch 1). The firing rate of the dACC neuron

192 was also affected by the expected value of the first offer when the second offer appeared

193 (epoch 2). This was not the case for the sgACC neuron.

194 Significant proportions of neurons in both regions encoded the value of the first

195 offer in the $500 \mathrm{~ms}$ epoch starting $100 \mathrm{~ms}$ after that offer appeared. Note that we chose

196 this epoch for another study and use it here to allow comparability across datasets (Strait

197 et al., 2014; Strait et al., 2015). 
$199=18 / 146)$ in sgACC were affected by the value of offer 1 during epoch 1 . Both

200 proportions are greater than chance (dACC: $\mathrm{P}<0.0001$; sgACC: $\mathrm{P}=0.0004$; two-sided

201 binomial test). Figure 3C shows the fraction of neurons whose firing rates were

202 modulated by offer 1 value through time. There was no measured bias towards a positive

203 direction in either area (see Table 3). The average regression weight across all neurons,

204 another measure of directionality of effect, was not different from zero in either area,

205 indicating no bias in the overall populations (see Table 2).

207 Neurons in both regions integrate gamble probability and stakes

208 The mathematical expected value of a gamble in our task - and its subjective

209 value - depend on three factors: the probability of the win, the win amount, and the loss

210 amount. We wanted to know whether the individual components of a gamble are

211 integrated into a single general value signal, or represented as separate attributes.

212 We explored whether probability and stakes were integrated in the first epoch of

213 the trial, when only the first gamble had been displayed. We compared regression

214 weights for neuronal responses to the first offer against the magnitude and probability of

215 a win and the magnitude of loss, in one multivariate regression, while controlling for

216 other variables that significantly explained firing rate for this neuron in this epoch (see

217 Methods for further detail).

218 Regression coefficients corresponding to win-magnitude and win-probability

219 were positively correlated, supporting the hypothesis that these parameters are integrated

220 This correlation is observed in both cingulate regions (dACC: $\mathrm{r}=0.63, \mathrm{P}<0.0001$; 
221 sgACC: $\mathrm{r}=0.31, \mathrm{P}=0.0001$; Pearson correlation coefficient of signed regression

222 coefficients). The absolute values of these coefficients were also positively correlated,

223 indicating that these variables were encoded by a single population of neurons rather than

224 two distinct ones - further supporting the integration hypothesis (dACC: $\mathrm{r}=0.59, \mathrm{P}<$

$2250.0001 ; \operatorname{sgACC}: \mathrm{r}=0.56, \mathrm{P}<0.0001)$. We previously reported similar findings in the

226 ventromedial prefrontal cortex (vmPFC) and the ventral striatum (VS, Strait et al., 2015).

227 These results suggest that all four regions carry integrated value signals.

228 Win and loss magnitudes were also integrated in both areas $(\mathrm{dACC}: \mathrm{r}=0.34, \mathrm{P}=$

$2290.0001 ; \operatorname{sgACC}: \mathrm{r}=0.36, \mathrm{P}<0.0001$; Pearson correlation coefficient of signed regression

230 coefficients). While these variables were represented in overlapping populations in

$231 \operatorname{sgACC}(\mathrm{r}=0.30, \mathrm{P}=0.0003$; Pearson correlation coefficient of unsigned regression

232 coefficients), this relationship does not achieve significance in $\mathrm{dACC}(\mathrm{r}=0.17, \mathrm{P}=$ $2330.053)$.

234

235 Neurons in both regions encode remembered values

236 We examined whether firing rates reflected the value of the first offer while the

237 second offer was presented (epoch 2, $500 \mathrm{~ms}$ epoch starting $100 \mathrm{~ms}$ after the second offer

238 appeared,). We saw encoding of offer 1 in this epoch in $\mathrm{dACC}(19.4 \%$ of neurons, $\mathrm{n}=$

$23925 / 129, \mathrm{P}<0.0001$; two-sided binomial test), but it failed to reach significance in sgACC

$240(8.22 \%, \mathrm{n}=12 / 146, \mathrm{P}=0.084)$. Since task-relevant signals were generally attenuated in

241 sgACC, and the percentage of tuned neurons we saw failed to reach significance by one

242 neuron, we repeated this analysis in an earlier $500 \mathrm{~ms}$ epoch, starting immediately when

243 the second offer appeared. The fraction of neurons tuned to the value of offer 1 in this 
244 epoch does achieve significance $(11.0 \%, \mathrm{n}=16 / 146, \mathrm{P}=0.0028$; two-sided binomial

245 test). This proportion is still significant when correcting for multiple comparisons (i.e. at

$246 \mathrm{P}<0.025)$. This finding suggests that sgACC may, in fact, carry a memory signal,

247 although one that is more attenuated and less reliable than that in dACC. There was no

248 bias in tuning in either brain region (see Table 3), nor was there any bias in the regression

249 coefficients of the overall populations (see Table 2).

250 We next investigated the relationship between the pattern of tuning for attended

251 and remembered offers. Was offer 1 encoded in similar tuning formats as it moved from

252 the retina to working memory? And were the putative working memory neurons, which

253 carried the value of offer 1 in the second epoch, the same ones that encoded it when it

254 was first presented - in the first epoch? The answers to both questions is yes for dACC,

255 but we cannot draw conclusions from our results in sgACC. We computed regression

256 weights for the expected value of offer 1 in epoch 1 (when it was present on the screen)

257 and for the same option in epoch 2 (when it was remembered), while controlling for other

258 task variables that best explained each neuron's response (see Methods). These values

259 were positively correlated in $\mathrm{dACC}(\mathrm{r}=0.32, \mathrm{P}=0.0002$; Pearson correlation coefficient

260 of signed regression coefficients), indicating a preservation of coding format as

261 information was transferred from an active to a remembered storage buffer. The absolute

262 regression coefficients were also positively correlated $(\mathrm{r}=0.44, \mathrm{P}<0.0001)$, suggesting

263 these variables were encoded by a single task-sensitive population, rather than memory

264 and active-buffer neurons (consistent with vmPFC and VS; Strait et al., 2015).

265 We cannot draw any strong conclusions from results in sgACC. There was no

266 significant correlation between these variables: neither one indicating preservation of 
267 format $(\mathrm{r}=0.090, \mathrm{P}=0.28$; Pearson correlation of signed regression coefficients), nor

268 one indicating that this signal was carried by the same population $(\mathrm{r}=0.0380, \mathrm{P}=0.65$;

269 Pearson correlation of unsigned regression coefficients). Results were similar in the

270 slightly earlier $500 \mathrm{~ms}$ epoch we used earlier (preservation of format: $\mathrm{r}=0.15, \mathrm{P}=0.079$;

271 overlapping populations: $\mathrm{r}=0.063, \mathrm{P}=0.45)$. It is difficult to draw conclusions from the

272 absence of this correlation, as it could be due to weaker signal-to-noise ratio in this

273 region. We therefore performed a cross-validation procedure similar to that in Blanchard

274 et al. (2015), and found that our signal in the epochs of interest was not strong enough to

275 detect correlations (see Methods). The failure of this procedure suggests that we could

276 not detect this effect with our data, even if it existed. We therefore draw no conclusions

277 from this null result.

278

A

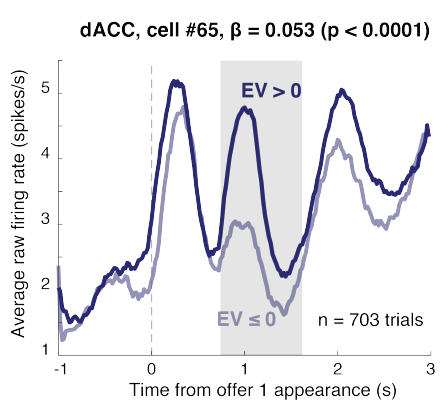

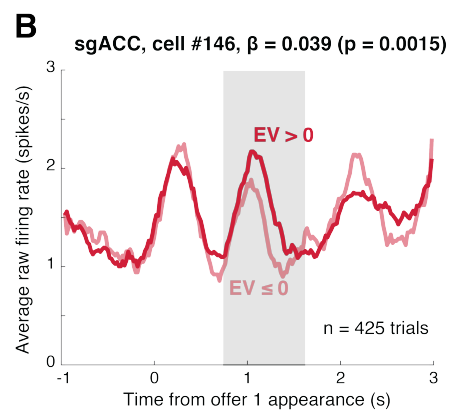

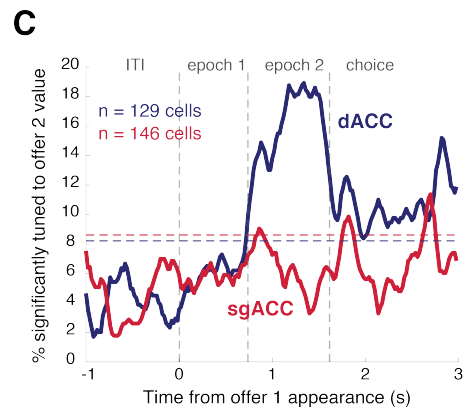

280 Figure 4: Neural responses to the value of offer 2. A: Responses of one dACC neuron whose firing rate increased in response to larger values of offer 2. B: Responses of one sgACC neuron whose firing rate increased in response to larger values of offer 2. C: Percentage of neurons tuned to the value of the second offer in both regions over the course of the trial. A $500 \mathrm{~ms}$ sliding window was used to compute these values at each point of the trial. The blue and red horizontal lines indicate the percentage of neurons we would expect to see tuned by chance (as determined by a two-sided binomial test, according to the number of neurons in each area) for $d A C C$ and $s g A C C$, respectively. 


\section{Simultaneous encoding of both offer values}

291 We next explored the effects of the second offer value on firing rates during

292 epoch 2. Figure 4 shows two example neurons, one from dACC and one from sgACC,

293 whose firing rates increased in response to the second offer. A significant proportion of

294 neurons in both regions encoded the value of the second offer when it appeared (dACC:

$29514.0 \%, \mathrm{n}=18 / 129 ; \operatorname{sgACC}: 9.59 \%, \mathrm{n}=14 / 146)$. Both proportions exceed what we would

296 predict by chance (dACC: $\mathrm{P}=0.0001$; sgACC: $\mathrm{P}=0.016$; two-sided binomial test).

297 There was no significant bias in tuning in either area (Table 3), and the average

298 coefficient of offer 2 value over the entire population was also not biased in either brain

299 region (Table 2). Figure 4C shows how the fraction of neurons responsive to offer 2

300 changed over the course of the trial.

301

\section{Attentionally-aligned coding scheme for offer values}

303 Do cingulate regions encode all attended offers similarly, using the same

304 population? Our previous studies of vmPFC and VS indicate that value coding in these

305 regions is attentionally-aligned - that is, they encode the value of the attended offer in a

306 conserved attended format (by format, we mean tuning strength and sign, and identity of

307 neurons; Strait et al., 2015; Akaishi \& Hayden, 2016; Rich \& Wallis, 2016).). This

308 framework differs from a labeled-line format, in which a specialized set of neurons

309 encodes the value of each offer respectively (e.g. Hunt et al., 2015). To test this, we

310 compared the regression coefficients associated with the first offer in the first epoch to

311 those associated with the second offer in the second epoch. 
312 Our data support the attentional alignment hypothesis. Specifically, coding

313 direction for both offers was consistent in both areas (dACC: $\mathrm{r}=0.51, \mathrm{P}<0.0001$;

314 sgACC: $\mathrm{r}=0.26, \mathrm{P}=0.0013$; Pearson correlation test of signed coefficients). Second,

315 both regions made use of a consistent set of neurons for the two offers (dACC: $r=0.45, P$

$316<0.0001$; sgACC: $\mathrm{r}=0.32, \mathrm{P}=0.0001$; Pearson correlation test of unsigned coefficients).

318 Beta anti-correlation in dACC: a putative signature of comparison through mutual

319 inhibition

320 One putative neural signature of the comparative process is an anti-correlation

321 between regression coefficients for the values of the two offers while they are being

322 compared (Strait et al., 2014). This anti-correlation indicates that the two offers modulate

323 neuronal activity in opposing directions, and that the ensemble of neurons effectively

324 subtracts their values. We observed this pattern in dACC during the second epoch — when

325 both offers had been presented $(r=-0.17, P=0.050$; Pearson correlation coefficient of

326 signed regression coefficients), suggesting that this area is involved in comparing the two

327 offers. We found no significant correlation in $\operatorname{sgACC}(r=-0.077, \mathrm{P}=0.35)$. It is difficult

328 to interpret the lack of a significant correlation as the absence of a comparison signal in

329 light of our weak signal-to-noise ratio, and due to the failure of the cross-validation

330 procedure (see Methods). 


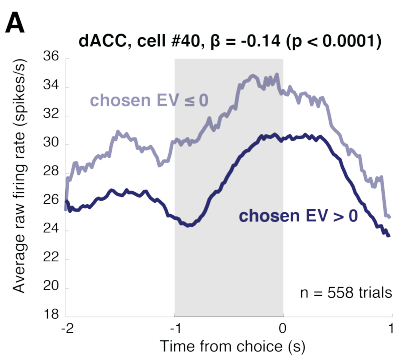

C

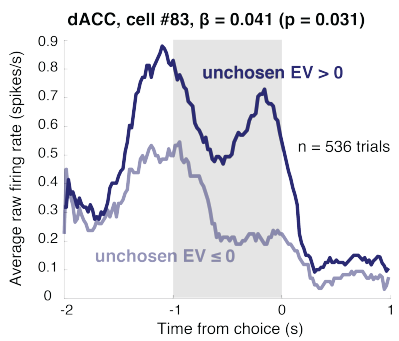

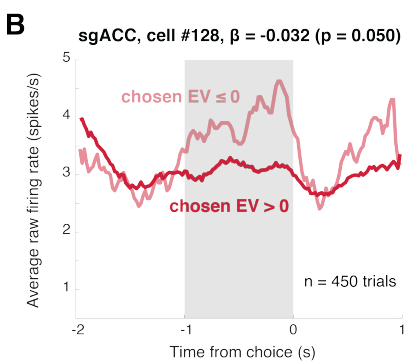

D

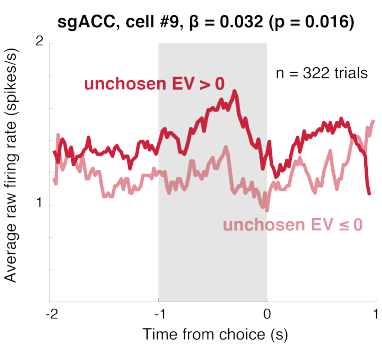

E

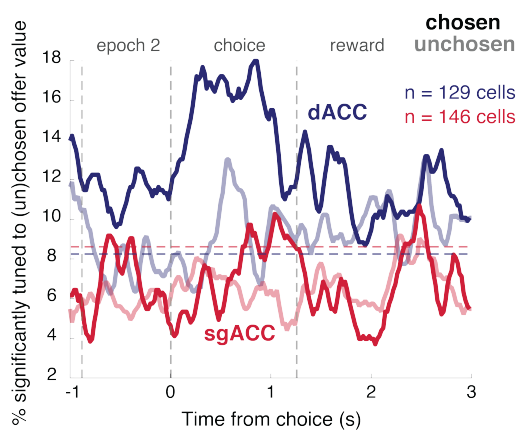

333 Figure 5: Neural responses to the values of the chosen and unchosen offers. A:

334 Responses of dACC neuron whose firing rate was negatively modulated by the value of

335 the chosen offer. This pattern is typical of neurons in this region (see text and Tables 2

336 and 3). B: Responses of sgACC neuron whose firing rate was negatively modulated by

337 the value of the chosen offer. $C$ : Responses of $d A C C$ neuron whose firing rate was

338 positively modulated by the value of the unchosen offer. This pattern is typical of neurons

339 in this region (see text and Tables 2 and 3). D: Responses of sgACC neuron whose firing

340 rate was positively modulated by the value of the unchosen offer. E: Percentage of

341 neurons tuned to the values of the chosen and unchosen offer values in both cingulate

342 regions throughout the course of the trial. Percentages were computed using a $500 \mathrm{~ms}$

343 sliding window, using a regression analysis (see Methods for details). The blue and red

344 horizontal lines indicate the percentage of neurons we would expect to see tuned by

345 chance (as determined by a two-sided binomial test, according to the number of neurons

346 in each area) for $d A C C$ and $s g A C C$, respectively.

348 Encoding of chosen and unchosen value signals in both regions

349 We examined selectivity for chosen and unchosen offer values in the 1-second

350 epoch ending with the saccade that indicated the subject's choice. We chose this epoch to

351 capture any correlates of the choice process that arose when the second offer was

352 presented (presumably when the comparison was made), and when the subject had to

353 indicate his choice. 
Figure 5 shows four neurons whose firing rates were affected by the values of the

355 chosen and unchosen offer values in both brain regions. Significant proportions of such

356 neurons were found in dACC, but not in sgACC. $15.5 \%(n=20 / 129)$ of dACC responded

357 to the chosen value (two-sided binomial test: $\mathrm{P}<0.0001)$, and $9.30 \%(\mathrm{n}=12 / 129)$

358 responded to the unchosen value $(\mathrm{P}=0.030)$. The proportion of neurons encoding chosen

359 value was greater than the proportion encoding unchosen value $(\mathrm{P}=0.018$; two-sided

360 binomial test). However, encoding of chosen value was not stronger than encoding of

361 unchosen value when the tuning coefficients of all neurons in the population were

362 compared $(\mathrm{T}=1.67, \mathrm{P}=0.097$; unpaired two-sample t-test of absolute regression

363 coefficients). There was more overlap than expected by chance between these

364 populations $(\mathrm{r}=0.22, \mathrm{P}=0.013$; Pearson correlation of unsigned regression coefficients $)$.

365 Figure 5E shows the change in these fractions as the choice was made.

366 There was a bias towards negative tuning for the chosen value in the tuned

367 population $(n=16 / 20$ negatively tuned, $P=0.012$; two-sided binomial test $)$, but not in

368 the regression coefficients of the overall population (Table 2). We found a bias towards

369 positive tuning for the unchosen value: both in the tuned population $(n=12 / 12$ positively

370 tuned; $\mathrm{P}=0.0005$; two-sided binomial test $)$, and in the overall population $(\mathrm{T}=2.70, \mathrm{P}=$

3710.0078 ; one-sample t-test).

372 No significant proportion of neurons encoding chosen $(6.16 \%, \mathrm{n}=9 / 146, \mathrm{P}=$

3730.57 ; two-sided binomial test $)$ or unchosen $(4.11 \%, \mathrm{n}=6 / 146, \mathrm{P}=0.71)$ offer values was

374 found in sgACC. However, the overall population was negatively biased towards

375 encoding of the chosen value $(\mathrm{T}=-2.31, \mathrm{P}=0.022$; one-sample t-test $)$, and exhibited a 
376 positive trend in encoding the unchosen value (Table 2), echoing the patterns we see in

377 dACC, albeit attenuated.

378

A

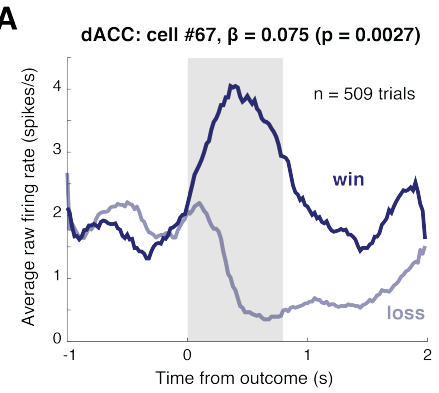

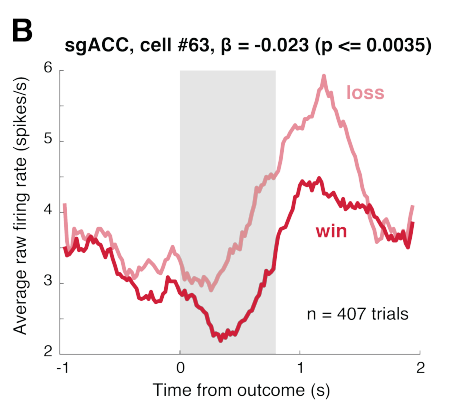

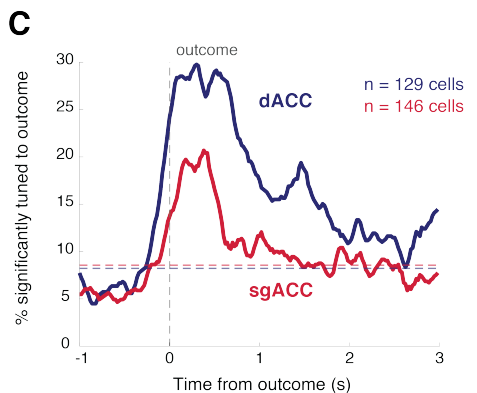

380 Figure 6: Neural responses to outcomes in both regions. A: Responses of dACC neuron with increasing firing rate in response to wins. There was a bias towards this pattern in the $d A C C$ population (see text and Table 2). B: sgACC neuron with increasing firing rate in response to losses. There was a bias towards this pattern in the sgACC population (see text and Table 2).

$C:$ Percentage of neurons in both regions tuned to the outcome of a trial throughout the course of a trial, using a $500 \mathrm{~ms}$ sliding window. The blue and red horizontal lines indicate the percentage of neurons we would expect to see tuned by chance (as determined by a two-sided binomial test, according to the number of neurons in each area) for $A A C C$ and $s g A C$, respectively.

\section{Encoding of gamble outcomes}

We examined selectivity for gamble outcomes during the $800 \mathrm{~ms}$ reward epoch that began with the reveal of the outcome and extended into the intertrial interval (see

393 Figure 1). Recall that outcomes in this task were tokens, not juice rewards. Figure 6

394 shows responses of two example neurons, one from each region, selective for outcome

395 amount. We excluded jackpot trials from this analysis so that liquid reward was matched

396 in all cases; the only difference was tokens given or taken. $24.03 \%(n=31 / 129)$ of neurons in $\mathrm{dACC}$ and $13.0 \%(\mathrm{n}=19 / 146)$ of neurons in $\mathrm{sgACC}$ encoded the outcome of

398 a trial. Both of these proportions are significant $(\mathrm{P}<0.0001$ in $\mathrm{dACC}$ and $\mathrm{P}=0.0001$ in sgACC; two-sided binomial test). Figure 6C shows the peak of these fractions in the 
400 outcome epoch. These are the first demonstration of $\operatorname{sgACC}$ selectivity to gains or losses

401 of secondary reinforcers.

402 Neurons in dACC showed a positive bias in outcome encoding. Specifically, the

403 majority of tuned neurons were positively tuned for outcome $(71.0 \%$ positively tuned, $\mathrm{n}=$

$40422 / 36, \mathrm{P}=0.029$; two-sided binomial test). There was also a positive bias in regression

405 coefficients in the overall population $(\mathrm{T}=2.35, \mathrm{P}=0.02$; one-sample t-test $)$. In sgACC,

406 neurons in the overall population were negatively tuned to outcomes $(\mathrm{T}=-2.85, \mathrm{P}=$

4070.0050 ; one-sample t-test), although this bias in the overall population's regression

408 weights was not significant in the tuned population (Table 3).

409
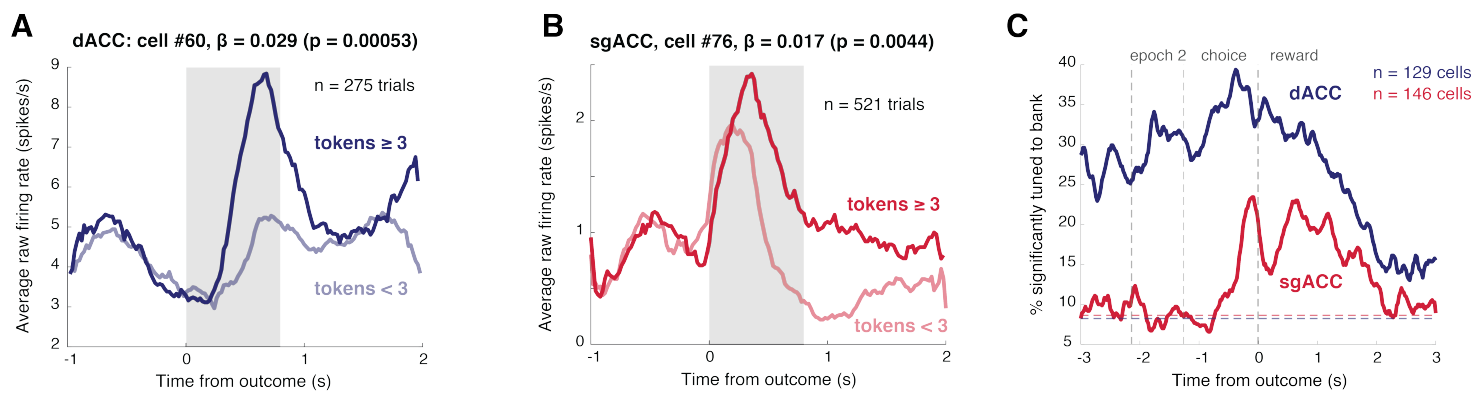

$411 \quad$ Figure 7: Neural responses to the number of tokens accumulated at the beginning of the

412 trial. A: Responses of dACC neuron that increased its firing rate as subjects accumulated

413 more tokens. B: Responses of sgACC neuron that increased its firing rate as subjects

414 accumulated more tokens. $C$ : Percentage of neurons in each area tuned to the number of

415 tokens accumulated throughout the course of the trial. All percentages were computed

416 using a $500 \mathrm{~ms}$ window (see Methods). The blue and red lines indicate the percentage of

417 neurons we would expect to see tuned by chance (as determined by a two-sided binomial

418 test, according to the number of neurons in each area) for $A A C C$ and sgACC,

419 respectively.

420

421 Both regions encode current number of tokens

422 In our task, the number of tokens provided a measure of progress towards the

423 jackpot reward. As noted, performance improved modestly with number of tokens. We 
424 hypothesized that this variable would affect neuronal responses in both cingulate areas.

425 Figure 7 shows two neurons (one in dACC, the other in sgACC) whose responses were

426 modulated by the number of tokens acquired during the 800 ms reward epoch. 34.9\% (n

$427=45 / 129)$ in $\mathrm{dACC}$ and $16.4 \%$ in $\operatorname{sgACC}(\mathrm{n}=24 / 146)$ significantly represented this

428 variable in that epoch. Both of these fractions are significant $(\mathrm{P}<0.0001$ in both regions;

429 two-sided binomial test). Figure $7 \mathrm{C}$ shows the change in this fraction throughout the trial.

430 There was no bias in tuning in either brain region: neither within the tuned population

431 (Table 3), nor in the overall population's regression coefficients (Table 2).

432

A

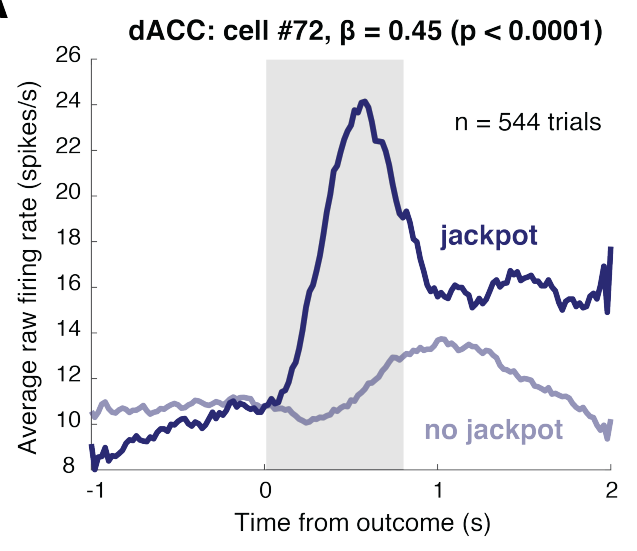

B

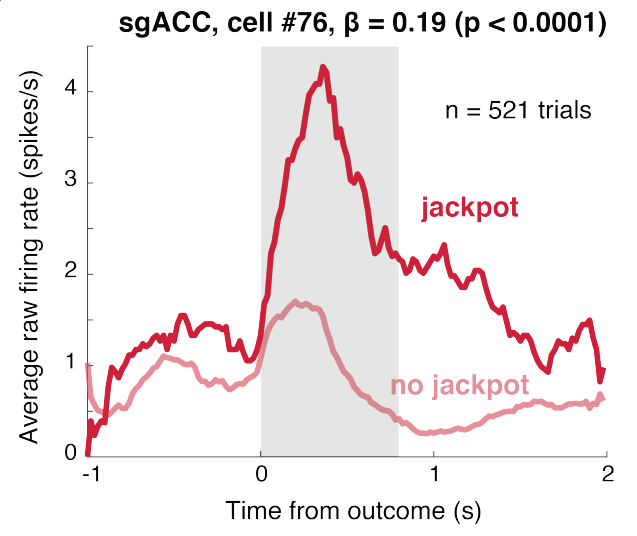

$434 \quad$ Figure 8: Neural responses to the anticipation of a large liquid reward (jackpot). A:

435 Responses of $d A C C$ neuron with increasing firing rate before jackpot rewards. B:

436 Responses of sgACC neuron with increasing firing rate before jackpot rewards. There

437 was a bias towards this response profile in the sgACC population (see text and Table 2).

\section{Neurons in both regions carry reward anticipation signals}

440 We quantified neural responses to anticipation of jackpots by comparing them to

441 responses on standard trials. Figure 8 shows two example neurons-one from each

442 region - with activity that varied with anticipation of a jackpot reward. Significant

443 proportions of such neurons were found in both regions $(27.3 \%$ in $\mathrm{dACC}, \mathrm{n}=35 / 129, \mathrm{P}<$ 
$444 \quad 0.0001 ; 11.6 \%$ in sgACC, $\mathrm{n}=17 / 146, \mathrm{P}=0.0011$; two-sided binomial test). We saw no

445 bias in positive or negative tuning in dACC: neither in the tuned population (Table 3), nor

446 in the population overall (Table 2). However, there was a positive bias in sgACC in the

447 overall population $(\mathrm{T}=4.47, \mathrm{P}<0.0001$; one-sample t-test), although not significantly in

448 the tuned population (Table 3).

\section{$450 \quad$ Neurons in both regions carry spatial signals}

451 We previously showed coding for two task variables, spatial position of offer and

452 chosen option, in both dACC and sgACC (Strait et al., 2016). The sgACC data used in

453 that study ( $\mathrm{n}=112$ neurons) were augmented with additional neurons collected in the

454 same animals in the same task ( $n=34$ additional neurons; total of 146 neurons). We still

455 find these effects in the larger dataset. (Note that for consistency with the other analyses

456 in this manuscript, we used a multiple linear regression analysis, see Methods).

457 Specifically, sgACC encoded the position of the offer during the first offer epoch $(\mathrm{n}=$

$45814 / 146$ neurons, $\mathrm{P}=0.016$; two-sided binomial test), and the position of the chosen

459 option during the post-choice epoch $(n=20 / 146$ neurons, $P<0.0001)$. Results remained

460 qualitatively similar in dACC with our new analyses. We see a significant proportion of

461 neurons tuned to the side of the first offer in the first epoch $(n=20 / 129$ neurons, $P<$

$4620.0001)$, and to the side of the chosen offer in the post-choice epoch $(n=30 / 129$ neurons,

$463 \quad \mathrm{P}<0.0001)$. 


\section{No significant difference in latency of encoding chosen side between regions}

468 We investigated latency differences between dACC and sgACC. We were

469 particularly interested in encoding of the chosen side, since this variable defined the

470 action ultimately taken to indicate the subject's decision. Conventional latency analyses

471 may be confounded due to the difference in signal-to-noise ratio between regions. This

472 problem is especially acute when these differences are large, as they are in our sgACC

473 and dACC datasets. In our data, signals are likely to achieve significance sooner in

474 dACC, simply because they are stronger than in sgACC.

475 To avoid this confound, we developed a new procedure to control for signal-to-

476 noise differences. We added Poisson distributed noise to our dACC dataset (mean =

477 mean firing rate of each cell), and tested the proportion of variance explained in this new

478 whitened dataset. If signal-to-noise in the reward epoch (when both regions were most

479 responsive) was within $1.5 \%$ of that in $\mathrm{sgACC}$, we included this dataset in our analyses.

480 We generated 500 datasets in this manner, then performed a latency analysis the same as

481 in Strait et al. (2015), with the slight modification that we used a multiple regression

482 model instead of a t-test to determine when neurons first responded significantly to

483 chosen side (this analysis takes into account neuronal responses to other variables, and is

484 therefore more robust). We used this manipulation to account for the variance explained

485 by other task-variables (listed in Methods). Even using this more sensitive measure and

486 better-controlled analysis, we failed to see a significant difference between latencies

487 across regions ( $t$-test across regions significant for $1 / 500$ datasets, $\mathrm{P}=0.998$ ). 


\section{DISCUSSION}

491 We examined responses of neurons in two cingulate regions, dACC and sgACC,

492 in a token gambling task. Both areas encoded several task variables, including values of

493 attended and remembered offers, outcomes, current token number, and, confirming an

494 earlier study, position of offered and chosen options. Neuronal response latencies were

495 similar in both areas. We observed two major differences between the regions: first, the

496 task-related signal-to-noise was consistently greater in AACC than in sgACC. Second, we

497 observed some differences in the average direction of value tuning: sgACC shows a

498 negative bias to outcomes while dACC shows a positive one, and, prior to jackpots,

499 sgACC shows a positive bias in activation, while dACC shows no consistent pattern.

500 Overall, these results point to a broad functional overlap between dACC and sgACC,

501 with differences more in emphasis than in major functional role.

502 Studies of functional neuroanatomy often focus on identifying each area's unique

503 specialization, but there are other important questions about brain regions - such as:

504 which regions subserve a given brain function? One mental operation may be

505 implemented by similar computations occurring simultaneously in multiple brain areas

506 (Behrmann \& Plaut, 2013; Cisek \& Kalaska, 2010; Cisek, 2012; Farah, 1994; McClelland

$507 \&$ Rumelhart, 1986). Our data suggest that risky economic choice may be mediated by

508 computations occurring in both dACC and sgACC, and, given our earlier data, across

509 vmPFC and VS as well (Strait et al., 2014; Strait et al., 2015). While these regions may,

510 in principle, compute similar variables for distinct purposes, it is more parsimonious to

511 assume, without strong evidence to the contrary, that they play similar roles. 
512 By way of analogy, an earlier generation of scholars proposed that early and mid-

513 level visual areas are specialized for specific aspects of form (Zeki, 1978), and that V4 is

514 specialized for color (Essen \& Zeki, 1978; Zeki, 1977; Zeki, 1980). Subsequent work

515 disproved this idea and demonstrated that its role in color processing is not very different

516 from those of adjacent areas (Schein et al., 1982; Motter, 1994; Van Essen \& Maunsell,

517 1983). Its properties are similar to (but slightly more complex than) its afferent V2 and

518 similar to (but slightly less complex than) its efferent PIT (Desimone \& Schein, 1987;

519 Desimone et al., 1985; Desimone \& Duncan, 1995; Hayden \& Gallant, 2013; Mirabella et

520 al., 2007). It would be a mistake to look for color vision in any one brain region: it

521 depends on the distributed and coordinated action of several brain regions, all of which

522 perform many other computations unrelated to color (Desimone \& Duncan, 1995;

523 Lennie, 1998). We suspect that the same principles apply to economic choice; it reflects

524 qualitatively similar, although quantitatively different, repeated simple computations

525 occurring in parallel across multiple prefrontal and striatal regions (and possibly others as

526 well). These results thus argue against a strong functional dichotomy between the two

527 regions. For example, Bush et al. (2000) argue that the dorsal cognitive region is

528 functionally distinct from the subgenual affective region and they may even inhibit each

529 other. We see no evidence for a cognitive/limbic split, nor of mutual inhibition between

530 these two regions. Clearly, other studies, using other methods, do see evidence for such a

531 split; further work will be required to reconcile these contradictions.

532 At the same time, the differences we observe between dACC and sgACC argue

533 against a mass action view of brain function. The differences we see are consistent with

534 results of both neuroimaging and lesion studies supporting some functional difference 
535 between the areas, and with the neuroanatomy, which shows different patterns of

536 connectivity (Heilbronner \& Haber, 2014 Vogt et al., 1995). The difference in signal-to-

537 noise is particularly intriguing. It may reflect a greater selectivity for choice tasks in the

538 dorsal region; presumably there are other tasks that would drive sgACC more effectively

539 than dACC (however, we would expect these differences to be quantitative, not

540 qualitative). Another possibility is that economic decision-making, while distributed, has

541 a fan-in structure and that dACC, which is presumably later in the hierarchy, has a more

542 concentrated and thus stronger set of task signals.

543 Our findings generally confirm and extend the existing literature on sgACC.

544 Monosov \& Hikosaka (2012), like us, find a bias towards encoding of negative outcomes

545 in area 25. We show that this finding extends to secondary (token) reinforcers. They

546 report no encoding of probability, and thus of integrated value; our finding of integrated

547 value encoding thus suggests that sgACC may play a more direct economic role,

548 especially in decision-making tasks. Amemori \& Graybiel (2012) recorded in pregenual

549 cingulum, in a region that is rostral to our region, but has a small overlap (see Methods).

550 They found a subzone, which may overlap with our subgenual recording site, biased

551 towards negative encodings. Our high fraction of subgenual neurons encoding upcoming

552 large rewards (jackpots) provide confirmation for the idea, proposed by Rudebeck and

553 colleagues (2014), that sgACC serves to sustain autonomic arousal in anticipation of a

554 reward. The phasic, task-relevant signals in sgACC, and its direct role in reward-

555 processing and anticipation, suggest that the role for subgenual neurons extends beyond

556 the control of basic arousal functions like sleep (Rolls et al., 2003). Our findings also

557 cohere with the findings relating activity in sgACC to negative affect and depression. 
558 Overactivity in this region correlates with depressive symptoms (Drevets, 2002; Mayberg

559 et al., 2000) and with transient sadness in healthy subjects (Mayberg et al., 1999); chronic

560 stimulation of sgACC can alleviate the symptoms of depression (Mayberg et al., 2005).

561 Negative mood inducing stimuli tend to activate sgACC (George et al., 1995; Mayberg et

562 al., 1997; Mayberg et al., 1999), and structural abnormalities in sgACC correlate with

563 mood disorders (Botteron et al., 2002; Coryell et al., 2005; Drevets et al., 1997).

564 These findings also have implications for our understanding of dACC as well.

565 The role of dACC in economic choice remains disputed. Some research indicates that it

566 serves as the site of choice (Rangel \& Hare, 2010); our work confirms this idea but, in the

567 broader context of data from other studies, suggests it does not play a unique role. Other

568 research suggests that dACC plays a specifically post-decisional role, in part because it

569 preferentially encodes the value of unchosen options (Blanchard \& Hayden, 2014;

570 Boorman et al., 2011; Rushworth et al., 2012; Hayden et al., 2009). Our results here

571 suggest that dACC may encode predecisional variables: the values of both chosen and

572 unchosen options - at least before the decision is made. Other work links dACC to

573 persistence - that is, in maintenance of value encoding until the time of reward to allow

574 stable behavior (Hillman \& Bilkey, 2013, Chudasama et al., 2013; Picton et al., 2007;

575 Blanchard et al., 2015; Shidara and Richmond, 2002). While we did not use a persistence

576 task, our results do endorse the idea that dACC serves to encode prospective rewards,

577 suggesting that it does serve this function although not necessarily purely for the purpose

578 of persistence.

579 Overall, these results invite speculation on whether one can ascribe a single

580 integrated role for the cingulum (Hayden \& Platt, 2009). On one hand, the broad overlap 
581 between $\mathrm{dACC}$ and sgACC that we see suggests it may have a general economic role. On

582 the other, our findings in a similar task in vmPFC and VS suggest this role may not be

583 unique to the cingulate cortex (Strait et al., 2014; Strait et al., 2015). Our results also have

584 some similarity to findings from the posterior cingulate cortex. Neural responses in that

585 region are associated with several economic variables, including offer and outcome

586 variables, as well as learning and control variables (Dean \& Platt, 2003; Hayden et al,

587 2009; Heilbronner \& Platt, 2013; McCoy et al., 2003; Heilbronner et al., 2011). Neurons

588 in sgACC tend to have poorer signal-to-noise than dACC, much like neurons in PCC

589 (Hayden et al., 2008), suggesting they may have some affinity. Still, we consider the

590 question of whether there is a general and unique function for the cingulum to be

591 unanswered.

592

\section{ACKNOWLEDGEMENTS}

594 We thank Caleb Strait for assistance designing the task, Tommy Blanchard, and

595 Alex Thomé for useful discussions, and Meghan Castagno, Giuliana Loconte and Marc

596 Mancarella for assistance in data collection. This research was supported by a NIH R01 


\section{MATERIALS AND METHODS}

605 Some of the data for both dACC and sgACC recordings were published (Strait et 606 al., 2016); all analyses presented here are new.

607

608 Surgical Procedures

609 All procedures were approved by the University Committee on Animal Resources

610 at the University of Rochester and were designed and conducted in compliance with the

611 Public Health Service's Guide for the Care and Use of Animals. Two male rhesus

612 macaques (Macaca mulatta: subject B age 5y. 7mo.; subject $\mathrm{J}$ age 6y. 7mo.) served as

613 subjects. A small prosthesis for holding the head was used. Animals were habituated to

614 laboratory conditions and then trained to perform oculomotor tasks for liquid reward. A

615 Cilux recording chamber (Crist Instruments) was placed over the dACC. Position was

616 verified by magnetic resonance imaging with the aid of a Brainsight system (Rogue

617 Research Inc.). Animals received appropriate analgesics and antibiotics after all

618 procedures. Throughout both behavioral and physiological recording sessions, the

619 chamber was kept sterile with regular antibiotic washes and sealed with sterile caps. All

620 recordings were performed during the animals' light cycle between $8 \mathrm{am}$ and 5pm.

621

\section{Recording Site}

623 We approached dACC through a standard recording grid (Crist Instruments). We

624 defined dACC according to the Paxinos atlas (Paxinos et al., 2000). Roughly, we

625 recorded from a ROI lying within the coronal planes situated between 29.50 and 34.50

$626 \mathrm{~mm}$ rostral to interaural plane, the horizontal planes situated between 4.12 to $7.52 \mathrm{~mm}$ 
627 from the brain's dorsal surface, and the sagittal planes between 0 and $5.24 \mathrm{~mm}$ from

628 medial wall. Our recordings were made from a central region within this zone. We

629 confirmed recording location before each recording session using our Brainsight system

630 with structural magnetic resonance images taken before the experiment. Neuroimaging

631 was performed at the Rochester Center for Brain Imaging, on a Siemens 3T

632 MAGNETOM Trio Tim using $0.5 \mathrm{~mm}$ voxels. We confirmed recording locations by

633 listening for characteristic sounds of white and gray matter during recording, which in all

634 cases matched the loci indicated by the Brainsight system.

635 We approached sgACC using similar equipment, in a similar manner. We defined

636 sgACC as lying within the coronal planes situated between 24 and $36 \mathrm{~mm}$ rostral to

637 interaural plane, the horizontal planes situated between 17.33 to $25.12 \mathrm{~mm}$ from the

638 brain's dorsal surface, and the sagittal planes between 0 and $5.38 \mathrm{~mm}$ from the medial

639 wall. Our recordings were made from a central region within this zone. We again confirm

640 recording locations using structural magnetic resonance images, Brainsight, and listening

641 to characteristic sounds of white and gray matter. These regions correspond to area 25 as

642 identified by Paxinos $(\mathrm{n}=118$ neurons) and also to the most caudal portion of area 32 ( $\mathrm{n}$

$643=28$ neurons).

644

645 Electrophysiological Techniques

646 Single electrodes (Frederick Haer \& Co., impedance range 0.8 to $4 \mathrm{MU}$ ) were

647 lowered using a microdrive (NAN Instruments) until waveforms of between one and

648 three neuron(s) were isolated. Individual action potentials were isolated on a Plexon

649 system (Plexon, Inc.). Neurons were selected for study solely on the basis of the quality 
650 of isolation; we never pre-selected based on task-related response properties. All

651 collected neurons for which we managed to obtain at least 250 trials were analyzed.

652

\section{Eye Tracking and Reward Delivery}

654 Eye position was sampled at $1,000 \mathrm{~Hz}$ by an infrared eye-monitoring camera

655 system (SR Research). Stimuli were controlled by a computer running Matlab

656 (Mathworks) with Psychtoolbox (Brainard,1997) and Eyelink Toolbox (Cornelissen et

657 al., 2002). Visual stimuli were colored rectangles on a computer monitor placed $57 \mathrm{~cm}$

658 from the animal and centered on its eyes (Figure 1A). A standard solenoid valve

659 controlled the duration of juice delivery. The relationship between solenoid open time

660 and juice volume was established and confirmed before, during, and after recording.

661

\section{Behavioral Task}

663 Monkeys performed a two-option gambling task. The task was similar to one we

664 have used previously (Strait et al., 2014; Strait et al., 2015), with two major differences:

665 (1) monkeys gambled for virtual tokens - rather than liquid — rewards, and (2) outcomes

666 could be losses as well as wins.

667 Two offers were presented on each trial. Each offer was represented by a rectangle 300

668 pixels tall and 80 pixels wide $\left(11.35^{\circ}\right.$ of visual angle tall and $4.08^{\circ}$ of visual angle wide).

$66920 \%$ of options were safe ( $100 \%$ probability of either 0 or 1 token), while the remaining

$67080 \%$ were gambles. Safe offers were entirely red ( 0 tokens) or blue (1 token). The size of

671 each portion indicated the probability of the respective reward. Each gamble rectangle

672 was divided horizontally into a top and bottom portion, each colored according to the 
673 token reward offered. Gamble offers were thus defined by three parameters: two possible

674 token outcomes, and probability of the top outcome (the probability of the bottom was

675 strictly determined by the probability of the top). The top outcome was $10 \%, 30 \%, 50 \%$,

$67670 \%$ or $90 \%$ likely.

677 Six initially unfilled circles arranged horizontally at the bottom of the screen

678 indicated the number of tokens to be collected before the subject obtained a liquid

679 reward. These circles were filled appropriately at the end of each trial, according to the

680 outcome of that trial. When 6 or more tokens were collected, the tokens were covered

681 with a solid rectangle while a liquid reward was delivered. Tokens beyond 6 did not carry

682 over, nor could number of tokens fall below zero.

683 On each trial, one offer appeared on the left side of the screen and the other

684 appeared on the right. Offers were separated from the fixation point by 550 pixels $\left(27.53^{\circ}\right.$

685 of visual angle). The side of the first offer (left and right) was randomized by trial. Each

686 offer appeared for $600 \mathrm{~ms}$ and was followed by a $150 \mathrm{~ms}$ blank period. Monkeys were

687 free to fixate upon the offers when they appeared (and in our observations almost always

688 did so). After the offers were presented separately, a central fixation spot appeared and

689 the monkey fixated on it for $100 \mathrm{~ms}$. Following this, both offers appeared simultaneously

690 and the animal indicated its choice by shifting gaze to its preferred offer and maintaining

691 fixation on it for $200 \mathrm{~ms}$. Failure to maintain gaze for $200 \mathrm{~ms}$ did not lead to the end of

692 the trial, but instead returned the monkey to a choice state; thus, monkeys were free to

693 change their mind if they did so within $200 \mathrm{~ms}$ (although in our observations, they

694 seldom did so). A successful $200 \mathrm{~ms}$ fixation was followed by a $750 \mathrm{~ms}$ delay, after

695 which the gamble was resolved and a small reward $(100 \mu \mathrm{L})$ was delivered - regardless 
696 of the outcome of the gamble - to sustain motivation. This small reward was delivered

697 within a $300 \mathrm{~ms}$ window. If 6 tokens were collected, a delay of $500 \mathrm{~ms}$ was followed by a

698 large liquid reward $(300 \mu \mathrm{L})$ within a $300 \mathrm{~ms}$ window, followed by a random inter-trial

699 interval (ITI) between 0.5 and 1.5 s. If 6 tokens were not collected, subjects proceeded

700 immediately to the ITI.

701 Each gamble included at least one positive or zero-outcome, ensuring that every

702 gamble carried the possibility of a win. This decreased the number of trivial choices

703 presented to subjects, and maintained motivation.

704

705 Statistical Methods

706 PSTHs were constructed by aligning spike rasters to the task event of interest

707 (offer 1 appearance, choice or feedback) and averaging firing rates across multiple trials.

708 Firing rates were calculated in $20 \mathrm{~ms}$ bins but were generally analyzed in longer (500-

$7091000 \mathrm{~ms}$ ) epochs. Plots were generated in Matlab, and use the Matlab function smooth

710 with a smoothing factor of 20.

711 Firing rates were normalized by subtracting the mean and dividing by the

712 standard deviation of the entire neuron's psth.

713 We test for significant tuning and assess variance explained using a multiple

714 linear regression, including the following task-relevant variables: expected value of

715 offers 1 and 2, the number of tokens collected as of the beginning of the trial, the side the

716 first offer appeared on, conflict between offer values (defined as the absolute difference

717 between them), the side of the chosen offer, the outcome of the trial (in tokens), and the

718 probability of that outcome (a measure of surprise). 
Analysis epochs were chosen a priori, before data analysis began, to reduce the

720 likelihood of p-hacking. The first and second offer epochs were defined as the $500 \mathrm{~ms}$

721 epoch beginning $100 \mathrm{~ms}$ after the offer was presented, to account for information

722 processing time. The choice epoch was the 1-second epoch before choice was indicated

723 using an express saccade. The reward epoch was defined as the $800 \mathrm{~ms}$ epoch following

724 the resolution of a gamble: this was when feedback and a small liquid reward were given

725 (regardless of trial outcome), followed by a $500 \mathrm{~ms}$ delay (this was part or all of the

726 intertrial interval on non-jackpot trials, and a pre-jackpot delay on jackpot trials).

727 All fractions of tuned neurons were tested for significance using a two-sided

728 binomial test. All binomial tests throughout the manuscript were two-sided.

729 All sliding window plots use a sliding $500 \mathrm{~ms}$ window, computing the fraction of

730 tuned neurons in the population every $20 \mathrm{~ms}$. Points on the plot are aligned to the start of

731 this window. Significance is assessed using a multiple linear regression including all

732 task-variables (mentioned above). Plots were smoothed using the Matlab function smooth

733 and a smoothing factor of 20.

734 We use beta correlation analyses using stepwise regression to assess whether

735 neurons represent two variables (or the same variable at different time periods) using

736 similar / orthogonal / opposing formats, in overlapping / orthogonal / distinct populations.

737 In these analyses, we first find the regression coefficients associated with the variables in

738 question, then find the Pearson correlation coefficient between these.

739 We noticed that, depending on which variables we included in the regression, the

740 results of our beta correlation analyses sometimes differed. We therefore used a stepwise

741 regression model as an objective method of determining which variables should be 
742 included in the final regression for each individual neuron. We first include all relevant

743 task variables, including the variables of interest, in this stepwise regression. We then

744 perform a (non-stepwise) multiple linear regression analysis again using only the

745 variables obtained from the previous step, as well as the variables of interest. We do this

746 to obtain a regression coefficient corresponding to the variable of interest for each

747 neuron, regardless of whether the coefficient for this variable achieved significance in the

748 previous step.

749 The Pearson correlation coefficient between signed regression coefficients

750 indicate whether variables were represented in a similar format i.e. directionality of

751 tuning across the population. A positive correlation indicates a preservation of

752 directionality, while a negative correlation indicates variables were represented in

753 opposing directionality of firing rate modulation. No correlation suggests orthogonal

754 formats, but we draw no strong conclusions from these.

755 Similarly, the Pearson correlation coefficient between unsigned regression

756 coefficients indicates whether similar neuronal populations tended to be involved in

757 encoding the two variables in question. A positive correlation indicates overlapping

758 populations, while a negative correlation indicates separate ones. A lack of correlation

759 suggests orthogonal populations (i.e. encoding one variable does not affect a neuron's

760 likelihood of encoding the other variable), but we again draw no strong conclusions from

761 this null result.

762 We performed a cross-validation procedure similar to that in Blanchard et al.

763 (Blanchard et al., 2015) on our sgACC dataset to determine whether our signal in the first

764 and second epochs was strong enough to detect correlations. We split trials into two 
765 random sets (even and odd), and computed the regression coefficients for each individual

766 neuron in response to offer 1 value in both epochs 1 and 2. We then obtained the Pearson

767 correlation coefficient of these signed regression coefficients within each epoch. We see

768 no correlation in the epoch we analyzed for offer 1 memory signals (i.e. epoch $2: \mathrm{r}=$

$7690.16, \mathrm{P}=0.058$, Pearson correlation coefficient). The failure of this cross-validation

770 procedure to detect a significant correlation in sgACC indicates that we did not have

771 sufficient signal in our dataset to detect correlation effects, even if they existed. This

772 procedure prevents us from drawing strong conclusions regarding the absence of a signal

773 in this region.

774

775 


\section{REFERENCES}

777

778

779

780

781

782

783

784

785

786

787

788

789

790

791

792

793

794

795

796

797

798

799

800

801

802

803

804

805

806

Akaishi, R., \& Hayden, B. Y. (2016). A Spotlight on Reward. Neuron, 90(6), 1148-1150. http://doi.org/10.1016/j.neuron.2016.06.008

Allman, J. M., Hakeem, A., Erwin, J. M., Nimchinsky, E., \& Hof, P. (2001). The Anterior Cingulate Cortex. Annals of the New York Academy of Sciences, 935(1), 107-117. http://doi.org/10.1111/j.1749-6632.2001.tb03476.x

Amemori, K. I., \& Graybiel, A. M. (2012). Localized microstimulation of primate pregenual cingulate cortex induces negative decision-making. Nature neuroscience, 15(5), 776-785.

Amiez, C., Joseph, J.-P., \& Procyk, E. (2005). Anterior cingulate error-related activity is modulated by predicted reward. European Journal of Neuroscience, 21(12), 34473452. http://doi.org/10.1111/j.1460-9568.2005.04170.x

Behrmann, M., \& Plaut, D. C. (2013). Distributed circuits, not circumscribed centers, mediate visual recognition. Trends in Cognitive Sciences, 17(5), 210-219. http://doi.org/10.1016/j.tics.2013.03.007

Blanchard, T. C., \& Hayden, B. Y. (2014). Neurons in Dorsal Anterior Cingulate Cortex Signal Postdecisional Variables in a Foraging Task. Journal of Neuroscience, 34(2), 646-655. http://doi.org/10.1523/JNEUROSCI.3151-13.2014

Blanchard, T. C., Hayden, B. Y., \& Bromberg-Martin, E. S. (2015). Orbitofrontal Cortex Uses Distinct Codes for Different Choice Attributes in Decisions Motivated by Curiosity. Neuron, 85(3), 602-614. http://doi.org/10.1016/j.neuron.2014.12.050

Blanchard, T. C., Strait, C. E., \& Hayden, B. Y. (2015). Ramping ensemble activity in dorsal anterior cingulate neurons during persistent commitment to a decision. Journal of Neurophysiology, 114(4), 2439-2449. http://doi.org/10.1152/jn.00711.2015

Blumberg, H. P., Stern, E., Martinez, D., Ricketts, S., De Asis, J., White, T., ... \& Silbersweig, D. A. (2000). Increased anterior cingulate and caudate activity in bipolar mania. Biological psychiatry, 48(11), 1045-1052.

Boorman ED, Behrens TE, Rushworth MF (2011) Counterfactual choice and learning in a neural network centered on human lateral frontopolar cortex. PLoS Biol 9:e1001093. CrossRef Medline 
807 Botteron, K. N., Raichle, M. E., Drevets, W. C., Heath, A. C., \& Todd, R. D. (2002).

808

809

810

811

812

813

814

815

816

817

818

819

820

821

822

823

824

825

826

827

828

829

830

831

832

833

834

835

836 Coryell, W., Nopoulos, P., Drevets, W., Wilson, T., \& Andreasen, N. C. (2005).

837

Volumetric reduction in left subgenual prefrontal cortex in early onset depression.

Biological Psychiatry, 51(4), 342-344. http://doi.org/10.1016/S00063223(01)01280-X

Bouras, C., Kövari, E., Hof, P. R., Riederer, B. M., \& Giannakopoulos, P. (2001).

Anterior cingulate cortex pathology in schizophrenia and bipolar disorder. Acta Neuropathologica, 102(4), 373-379. http://doi.org/10.1007/s004010100392

Bush, G., Frazier, J. A., Rauch, S. L., Seidman, L. J., Whalen, P. J., Jenike, M. A., et al. (1999). Anterior cingulate cortex dysfunction in attention-deficit/hyperactivity disorder revealed by fMRI and the counting stroop. Biological Psychiatry, 45(12), 1542-1552. http://doi.org/10.1016/S0006-3223(99)00083-9

Bush, G., Luu, P., \& Posner, M. I. (2000). Cognitive and emotional influences in anterior cingulate cortex. Trends in Cognitive Sciences, 4(6), 215-222. http://doi.org/10.1016/S1364-6613(00)01483-2

Bush, G., Vogt, B. A., Holmes, J., Dale, A. M., Greve, D., Jenike, M. A., \& Rosen, B. R. (2002). Dorsal anterior cingulate cortex: a role in reward-based decision making. Proceedings of the National Academy of Sciences, 99(1), 523-528. http://doi.org/10.1073/pnas.012470999

Carter, C. S., \& van Veen, V. (2007). Anterior cingulate cortex and conflict detection: An update of theory and data. Cognitive, Affective, \& Behavioral Neuroscience, 7(4), 367-379. http://doi.org/10.3758/CABN.7.4.367

Chudasama, Y., Daniels, T. E., Gorrin, D. P., Rhodes, S. E., Rudebeck, P. H., \& Murray, E. A. (2013). The role of the anterior cingulate cortex in choices based on reward value and reward contingency. Cerebral Cortex, 23(12), 2884-2898.

Cisek, P. (2012). Making decisions through a distributed consensus. Current Opinion in Neurobiology, 22(6), 927-936. http://doi.org/10.1016/j.conb.2012.05.007

Cisek, P., \& Kalaska, J. F. (2010). Neural Mechanisms for Interacting with a World Full of Action Choices. Dx.Doi.org, 33(1), 269-298.

http://doi.org/10.1146/annurev.neuro.051508.135409

Subgenual Prefrontal Cortex Volumes in Major Depressive Disorder and 

Journal of Psychiatry, 162(9), 1706-1712.

841 Cotter, D., Mackay, D., Landau, S., Kerwin, R., \& Everall, I. (2001). Reduced Glial Cell Density and Neuronal Size in the Anterior Cingulate Cortex in Major Depressive Disorder. Archives of General Psychiatry, 58(6), 545-553. http://doi.org/10.1001/archpsyc.58.6.545

845 Dean, H. L., \& Platt, M. L. (2003). Spatial representations in posterior cingulate cortex. Journal of Vision, 3(9), 427-427. http://doi.org/10.1167/3.9.427

847 Derbyshire, S. W. G., Vogt, B. A., \& Jones, A. K. P. (1998). Pain and Stroop interference tasks activate separate processing modules in anterior cingulate cortex. Experimental Brain Research, 118(1), 52-60. http://doi.org/10.1007/s002210050254

Desimone, R., \& Duncan, J. (1995). Neural mechanisms of selective visual attention.

853 Desimone, R., \& Schein, S. J. (1987). Visual properties of neurons in area V4 of the macaque: sensitivity to stimulus form. Journal of Neurophysiology, 57(3), 835-

856 Desimone, R., Schein, S. J., Moran, J., \& Ungerleider, L. G. (1985). Contour, color and shape analysis beyond the striate cortex. Vision Research, 25(3), 441-452.

859 Drevets, W. (2002). Functional anatomical correlates of antidepressant drug treatment assessed using PET measures of regional glucose metabolism. European

863 Drevets, W. C., Price, J. L., Simpson, J. R., Todd, R. D., Reich, T., Vannier, M., \& Raichle, M. E. (1997). Subgenual prefrontal cortex abnormalities in mood

866 Drevets, W. C., Savitz, J., \& Trimble, M. (2008). The subgenual anterior cingulate cortex 867 in mood disorders. CNS Spectrums, 13(8), 663-681.

868 Dum, R. P., Levinthal, D. J., \& Strick, P. L. (2016). Motor, cognitive, and affective areas 
869

870

871

872

873

874

875

876

877

878

879

880

881

882

883

884

885

886

887

888

889

890

891

892

893

894

895

896

897

898

899

of the cerebral cortex influence the adrenal medulla. Proceedings of the National Academy of Sciences of the United States of America, 113(35), 9922-9927. http://doi.org/10.1073/pnas.1605044113

Essen, D. C., \& Zeki, S. M. (1978). The topographic organization of rhesus monkey prestriate cortex. The Journal of Physiology, 277, 193-226. http://doi.org/10.1111/(ISSN)1469-7793

Farah, M. J. (1994). Neuropsychological inference with an interactive brain: A critique of the "locality" assumption. Behavioral and Brain Sciences, 17(1), 43-61. http://doi.org/10.1017/S0140525X00033306

Forman, S. D., Dougherty, G. G., Casey, B. J., Siegle, G. J., Braver, T. S., Barch, D. M., ... \& Lorensen, E. (2004). Opiate addicts lack error-dependent activation of rostral anterior cingulate. Biological psychiatry, 55(5), 531-537.

Freedman, L. J., Insel, T. R., \& Smith, Y. (2000). Subcortical projections of area 25 (subgenual cortex) of the macaque monkey. Journal of Comparative Neurology, 421(2), 172-188. http://doi.org/10.1002/(SICI)10969861(20000529)421:2<172::AID-CNE4>3.0.CO;2-8

George, M. S., Ketter, T. A., Parekh, P. I., Horwitz, B., Herscovitch, P., \& Post, R. M. (2006). Brain activity during transient sadness and happiness in healthy women. American Journal of Psychiatry, 152(3), 341-351. http://doi.org/10.1176/ajp.152.3.341

Goldstein, R. Z., Tomasi, D., Rajaram, S., Cottone, L. A., Zhang, L., Maloney, T., et al. (2007). Role of the anterior cingulate and medial orbitofrontal cortex in processing drug cues in cocaine addiction. Neuroscience, 144(4), 1153-1159. http://doi.org/10.1016/j.neuroscience.2006.11.024

Graybiel, A. M., \& Rauch, S. L. (2000). Toward a Neurobiology of ObsessiveCompulsive Disorder. Neuron, 28(2), 343-347. http://doi.org/10.1016/S08966273(00)00113-6

Hayden, B., \& Gallant, J. (2013). Working Memory and Decision Processes in Visual Area V4. Frontiers in Neuroscience, 7. http://doi.org/10.3389/fnins.2013.00018

Hayden, B. Y., Heilbronner, S. R., Nair, A. C., \& Platt, M. L. (2008). Cognitive influences on risk-seeking by rhesus macaques. Judgment and Decision Making, 
3(5), 389-395.

901 Hayden, B. Y., Nair, A. C., McCoy, A. N., \& Platt, M. L. (2008). Posterior Cingulate

902 Cortex Mediates Outcome-Contingent Allocation of Behavior. Neuron, 60(1), 19-

903 25. http://doi.org/10.1016/j.neuron.2008.09.012

904 Hayden, B. Y., Pearson, J. M., \& Platt, M. L. (2009). Fictive reward signals in the

905 anterior cingulate cortex. science, 324(5929), 948-950.

906 Hayden, B. Y., Pearson, J. M., \& Platt, M. L. (2011). Neuronal basis of sequential

907 foraging decisions in a patchy environment. Nature neuroscience, 14(7), 933-939.

908 Hayden, B. Y., \& Platt, M. L. (2007). Temporal Discounting Predicts Risk Sensitivity in

909 Rhesus Macaques. Current Biology, 17(1), 49-53.

$910 \quad$ http://doi.org/10.1016/j.cub.2006.10.055

911 Hayden, B. Y., \& Platt, M. L. (2009). Cingulate cortex. Encyclopaedia of Neuroscience.

$912 \quad$ http://doi.org/10.3389/fnhum.2012.00124/abstract

913 Hayden, B. Y., Smith, D. V., \& Platt, M. L. (2009). Electrophysiological correlates of

914 default-mode processing in macaque posterior cingulate cortex. Proceedings of the

$915 \quad$ National Academy of Sciences of the United States of America, 106(14), 5948-

916 5953. http://doi.org/10.1073/pnas.0812035106

917 Heilbronner, S. R., \& Haber, S. N. (2014). Frontal cortical and subcortical projections

918 provide a basis for segmenting the cingulum bundle: implications for

919 neuroimaging and psychiatric disorders. The Journal of Neuroscience, 34(30),

$920 \quad 10041-10054$.

921 Heilbronner, S. R., \& Hayden, B. (2015). Contextual factors explain risk-seeking

922 preferences in rhesus monkeys. Decision Making under Uncertainty, 91.

923 Heilbronner, S. R., \& Hayden, B. Y. (2016). Dorsal Anterior Cingulate Cortex: A

924 Bottom-Up View. Annual Review of Neuroscience.

925 Heilbronner, S. R., \& Platt, M. L. (2013). Causal Evidence of Performance Monitoring

926 by Neurons in Posterior Cingulate Cortex during Learning. Neuron, 80(6), 1384-

927 1391. http://doi.org/10.1016/j.neuron.2013.09.028

928 Hillman, K. L., \& Bilkey, D. K. (2013). Persisting through subjective effort: A key role

929 for the anterior cingulate cortex? Behavioral and Brain Sciences, 36(6), 691-692.

$930 \quad$ http://doi.org/10.1017/S0140525X13001039 
931 Hosokawa, T., Kennerley, S. W., Sloan, J., \& Wallis, J. D. (2013). Single-neuron

932

933

934

935

936

937

938

939

940

941

942

943

944

945

946

947

948

949

950

951

952

953

954

955

956

957

958

959

960

961 mechanisms underlying cost-benefit analysis in frontal cortex. The Journal of Neuroscience, 33(44), 17385-17397. http://doi.org/10.1523/JNEUROSCI.222113.2013

Hunt, L. T., Behrens, T. E., Hosokawa, T., Wallis, J. D., \& Kennerley, S. W. (2015). Capturing the temporal evolution of choice across prefrontal cortex. Elife, 4, e11945.

Johansen-Berg, H., Gutman, D. A., Behrens, T. E. J., Matthews, P. M., Rushworth, M. F. S., Katz, E., et al. (2008). Anatomical Connectivity of the Subgenual Cingulate Region Targeted with Deep Brain Stimulation for Treatment-Resistant Depression. Cerebral Cortex, 18(6), 1374-1383. http://doi.org/10.1093/cercor/bhm167

Kennerley, S. W., Walton, M. E., Behrens, T. E. J., Buckley, M. J., \& Rushworth, M. F. S. (2006). Optimal decision making and the anterior cingulate cortex. Nature Neuroscience, 9(7), 940-947. http://doi.org/10.1038/nn1724

Kerns, J. G., Cohen, J. D., MacDonald, A. W., Cho, R. Y., Stenger, V. A., \& Carter, C. S. (2004). Anterior Cingulate Conflict Monitoring and Adjustments in Control. Science, 303(5660), 1023-1026. http://doi.org/10.1126/science.1089910

Lak, A., Stauffer, W. R., \& Schultz, W. (2014). Dopamine prediction error responses integrate subjective value from different reward dimensions. Proceedings of the National Academy of Sciences of the United States of America, 111(6), 2343-2348. http://doi.org/10.1073/pnas.1321596111

Lennie, P. (1998). Single Units and Visual Cortical Organization. Perception, 27(8), 889-935. http://doi.org/10.1068/p270889

Mayberg, H. S., Brannan, S. K., Mahurin, R. K., Jerabek, P. A., Brickman, J. S., Tekell, J. L., et al. (1997). Cingulate function in depression: a potential predictor of treatment response. NeuroReport, 8(4), 1057.

Mayberg, H. S., Brannan, S. K., Tekell, J. L., Silva, J. A., Mahurin, R. K., McGinnis, S., \& Jerabek, P. A. (2000). Regional metabolic effects of fluoxetine in major depression: serial changes and relationship to clinical response. Biological Psychiatry, 48(8), 830-843. http://doi.org/10.1016/S0006-3223(00)01036-2 Mayberg, H. S., Liotti, M., \& Brannan, S. K. (1999). Reciprocal limbic-cortical function 
and negative mood: converging PET findings in depression and normal sadness. American Journal of ....

964 Mayberg, H. S., Lozano, A. M., Voon, V., McNeely, H. E., Seminowicz, D., Hamani, C., 965 et al. (2005). Deep Brain Stimulation for Treatment-Resistant Depression. Neuron, 45(5), 651-660. http://doi.org/10.1016/j.neuron.2005.02.014

967 McClelland, J. L., Rumelhart, D. E., \& Hinton, G. E. (1986). The appeal of parallel

968 distributed processing. MIT Press, Cambridge MA, 3-44.

969 McCoy, A. N., Crowley, J. C., Haghighian, G., Dean, H. L., \& Platt, M. L. (2003).

970 Saccade Reward Signals in Posterior Cingulate Cortex. Neuron, 40(5), 1031-1040.

971 http://doi.org/10.1016/S0896-6273(03)00719-0

972 McCoy, A. N., \& Platt, M. L. (2005). Risk-sensitive neurons in macaque posterior

973 cingulate cortex. Nature neuroscience, 8(9), 1220-1227.

974 Mirabella, G., Bertini, G., Samengo, I., Kilavik, B. E., Frilli, D., Libera, Della, C., \&

975 Chelazzi, L. (2007). Neurons in Area V4 of the Macaque Translate Attended Visual Features into Behaviorally Relevant Categories. Neuron, 54(2), 303-318.

978 Monosov, I. E., \& Hikosaka, O. (2012). Regionally distinct processing of rewards and punishments by the primate ventromedial prefrontal cortex. The Journal of Neuroscience, 32(30), 10318-10330. http://doi.org/10.1523/JNEUROSCI.180112.2012

Motter, B. C. (1994). Neural correlates of attentive selection for color or luminance in

984 Neubert, F.-X., Mars, R. B., Sallet, J., \& Rushworth, M. F. S. (2015). Connectivity reveals relationship of brain areas for reward-guided learning and decision making in human and monkey frontal cortex. Proceedings of the National Academy of

988 Öngür, D., Drevets, W. C., \& Price, J. L. (1998). Glial reduction in the subgenual prefrontal cortex in mood disorders. Proceedings of the National Academy of Sciences, 95(22), 13290-13295. http://doi.org/10.1073/pnas.95.22.13290

991 Paus, T. (2001). Primate Anterior Cingulate Cortex: Where Motor Control, Drive and Cognition Interface. Nature Reviews Neuroscience, 1-8. 
993 Picton, T. W., Stuss, D. T., Alexander, M. P., Shallice, T., Binns, M. A., \& Gillingham, S. (2007). Effects of focal frontal lesions on response inhibition. Cerebral Cortex, $17(4), 826-838$.

996 Procyk, E., Tanaka, Y. L., \& Joseph, J. P. (2000). Anterior cingulate activity during routine and non-routine sequential behaviors in macaques. Nature Neuroscience, 3(5), 502-508. http://doi.org/10.1038/74880

999 Quilodran, R., Rothé, M., \& Procyk, E. (2008). Behavioral Shifts and Action Valuation in the Anterior Cingulate Cortex. Neuron, 57(2), 314-325. http://doi.org/10.1016/j.neuron.2007.11.031

Rangel, A., \& Hare, T. (2010). Neural computations associated with goal-directed choice. Current Opinion in Neurobiology, 20(2), 262-270. http://doi.org/10.1016/j.conb.2010.03.001

Rich, E. L., \& Wallis, J. D. (2016). Decoding subjective decisions from orbitofrontal cortex. Nature Neuroscience, 19(7), 973-980. http://doi.org/10.1038/nn.4320

Rolls, E. T., Inoue, K., \& Browning, A. (2003). Activity of primate subgenual cingulate cortex neurons is related to sleep. Journal of Neurophysiology, 90(1), 134-142.

Rudebeck, P. H., Putnam, P. T., Daniels, T. E., Yang, T., Mitz, A. R., Rhodes, S. E., \& Murray, E. A. (2014). A role for primate subgenual cingulate cortex in sustaining autonomic arousal. Proceedings of the National Academy of Sciences, 111(14), 5391-5396.

Rushworth, M. F. S., Hadland, K. A., Paus, T., \& Sipila, P. K. (2002). Role of the Human Medial Frontal Cortex in Task Switching: A Combined fMRI and TMS Study. Journal of Neurophysiology, 87(5), 2577-2592. http://doi.org/10.1016/S00283932(97)00003-1

Schein, S. J., Marrocco, R. T., \& De Monasterio, F. M. (1982). Is there a high concentration of color-selective cells in area V4 of monkey visual cortex? Journal

1023 Seo, H., \& Lee, D. (2007). Temporal filtering of reward signals in the dorsal anterior 
1024

1025

1026

1027

1028

1029

1030

1031

1032

1033

1034

1035

1036

1037

1038

1039

1040

1041

1042

1043

1044

1045

1046

1047

1048

1049

1050

1051

1052

1053

1054

cingulate cortex during a mixed-strategy game. The Journal of Neuroscience.

Shenhav, A., Botvinick, M. M., \& Cohen, J. D. (2013). The Expected Value of Control: An Integrative Theory of Anterior Cingulate Cortex Function. Neuron, 79(2), 217240. http://doi.org/10.1016/j.neuron.2013.07.007

Shidara, M., \& Richmond, B. J. (2002). Anterior Cingulate: Single Neuronal Signals Related to Degree of Reward Expectancy. Science, 296(5573), 1709-1711. http://doi.org/10.1126/science.1069504

Strait, C. E., Blanchard, T. C., \& Hayden, B. Y. (2014). Reward Value Comparison via Mutual Inhibition in Ventromedial Prefrontal Cortex. Neuron, 82(6), 1357-1366. http://doi.org/10.1016/j.neuron.2014.04.032

Strait, C. E., Sleezer, B. J., Blanchard, T. C., Azab, H., Castagno, M. D., \& Hayden, B. Y. (2016). Neuronal selectivity for spatial positions of offers and choices in five reward regions. Journal of neurophysiology, 115(3), 1098-1111.

Strait, C. E., Sleezer, B. J., \& Hayden, B. Y. (2015). Signatures of Value Comparison in Ventral Striatum Neurons. PLoS Biol, 13(6), e1002173. http://doi.org/10.1371/journal.pbio.1002173

Van Essen, D. C., \& Maunsell, J. H. R. (1983). Hierarchical organization and functional streams in the visual cortex. Trends in Neurosciences, 6, 370-375. http://doi.org/10.1016/0166-2236(83)90167-4

Vogt, B. A., Finch, D. M., \& Olson, C. R. (1992). Functional Heterogeneity in Cingulate Cortex: The Anterior Executive and Posterior Evaluative Regions. Cerebral Cortex, 2(6), 435-443. http://doi.org/10.1093/cercor/2.6.435-a

Vogt, B. A., Nimchinsky, E. A., Vogt, L. J., \& Hof, P. R. (1995). Human cingulate cortex: Surface features, flat maps, and cytoarchitecture. Journal of Comparative Neurology, 359(3), 490-506. http://doi.org/10.1002/cne.903590310

Wallis, J. D., \& Kennerley, S. W. (2010). Heterogeneous reward signals in prefrontal cortex. Current Opinion in Neurobiology, 20(2), 191-198. http://doi.org/10.1016/j.conb.2010.02.009

Yamada, H., Tymula, A., Louie, K., \& Glimcher, P. W. (2013). Thirst-dependent risk preferences in monkeys identify a primitive form of wealth. Proceedings of the National Academy of Sciences of the United States of America, 110(39), 15788- 
15793. http://doi.org/10.1073/pnas.1308718110

1056 Zeki, S. M. (1977). Colour Coding in the Superior Temporal Sulcus of Rhesus Monkey

1057 Visual Cortex. Proceedings of the Royal Society B: Biological Sciences,

1058 197(1127), 195-223. http://doi.org/10.1098/rspb.1977.0065

1059 Zeki, S. M. (1978). Functional specialisation in the visual cortex of the rhesus monkey.

$1060 \quad$ Nature, 274(5670), 423-428.

1061 Zeki, S. (1980). The representation of colours. Nature, 284, 413.

1062 